\title{
SI units in pathology: the next stage
}

Three yeais ago recommendations were made for the first stage of the introduction of the Systéme International d'Unités (SI) into pathology in Britain (Journal of Clinical Pathology, 1970, 23, 818-819). These emphasized the need for the correct and unambiguous use of various units and their symbols (Units, Symbols and Abbreviations, 1973, Royal Society of Medicine), and introduced new units of the mole (symbol: mol) for amount of substance and the pascal (symbol: $\mathrm{Pa}$ ) for pressure. It was proposed that all pathology laboratories should act on these recommendations. However, it was made clear that the mole, for the present, should only be used replacing the equivalent for monovalent ions such as sodium $\left(\mathrm{Na}^{+}\right)$where the results would be numerically identical. This course of action has been generally adopted.

Over the last two years a working party of representatives of many relevant laboratory-based organizations has been considering the second stage in the introduction of SI. The recommendations will refer to all laboratory and measuring sciences related to medicine, for example, to medical physics as well as to pathology. The main change will be the use of the mole and molar concentration for all substances of known chemical structure and molecular weight and not only for monovalent ions, and this will mean some initially unfamiliar results, particularly in chemical pathology and haematology. In addition the pascal will become the preferred unit of pressure. The joule (symbol: J) will be used for all forms of energy, replacing the calorie for heat energy as already accepted by nutritional scientists. This policy will keep pathology in line with other branches of science and technology.

The litre will be used as the preferred volume unit for particle counts and for mass concentration in the same way as it is used for molar concentration. The litre replaces for mass concentration the unit of $100 \mathrm{ml}$, otherwise known as the decilitre (symbol: $\mathrm{dl}$ ).

In haematology it has been decided that mass concentration should be maintained for proteins (eg, haemoglobin, transferrin) and for other substances (vitamin $B_{12}$, folate) where the molecular weight of the biologically active entity is uncertain, so the use of molar concentration is impracticable. To avoid confusion in medical practice throughout the world the decilitre volume unit may be retained for haemoglobin only, so a normal blood haemoglobin concentration may be expressed as $14 \mathrm{~g} / \mathrm{dl}$ or $140 \mathrm{~g} / \mathrm{l}$ : in Britain the former is recommended.

In chemical pathology similarly mass concentration will be maintained for all proteins, but expressed per litre. For example, a normal serum albumin would be $45 \mathrm{~g} / \mathrm{l}$. For all other substances of known molecular weight, whether organic or inorganic, molar concentration will be used. For example, a serum sodium of $150 \mathrm{~m}$-equiv/ 1 becomes $150 \mathrm{mmol} / \mathrm{l}$ (a change already accepted), a serum calcium of $10 \mathrm{mg} / 100 \mathrm{ml}$ becomes $2.5 \mathrm{mmol} / 1$, a plasma glucose of $90 \mathrm{mg} / 100 \mathrm{ml}$ becomes $5.0 \mathrm{mmol} / 1$. Different base units, for example, time as $\mathbf{2 4}$ hours, apply to excretion rates.

Special non-SI units may be employed for special proteins such as enzymes and immunoglobulins. A new unit which is compatible with SI, the katal 
(symbol: kat), has been proposed for enzyme activity: this is the catalytic amount that transforms 1 mole of substrate per second under defined conditions. It has not yet been universally accepted, and the working party are making no recommendation regarding enzyme units.

These recommendations on SI units are in accord with those made jointly in 1972 by the International Federation of Clinical Chemistry, the International Committee for Standardization in Hematology, and the World Association of Pathology Societies. Before they can be implemented, discussions will take place with user organizations (eg, physicians, pharmacists, nurses) on the necessary educational and practical steps and on possible clarifications. A detailed document will then be prepared discussing SI in relation to pathology, and listing normal values in both traditional and SI units and conversion factors for haematology and chemical pathology. Many scientific journals and hospital laboratories are already using this system. The working party hope that it will be possible for the majority of hospital laboratories in Britain to have made the changes by the end of 1974.

D. N. BARON 